\section{Disponibilidade e consumo de ultraprocessados em escolas do Município de São Paulo, Brasil: resultados do SP-Proso}

\author{
Availability and consumption of ultra-processed \\ foods in schools in the municipality of São Paulo, \\ Brazil: results of the SP-Proso
}

\section{Disponibilidad y consumo de ultraprocesados en escuelas del Municipio de São Paulo, Brasil: resultados del SP-Proso}

Maria Alvim Leite 1

Catarina Machado Azeredo 2

Maria Fernanda Tourinho Peres 1

Maria Mercedes Loureiro Escuder 3

Renata Bertazzi Levy 1

\title{
Resumo
}

Alimentos ultraprocessados são marcadores de padrões alimentares não saudáveis e seu consumo é elevado entre os adolescentes. Características do ambiente alimentar escolar se associam a práticas alimentares de estudantes. $O$ objetivo do estudo foi investigar a associação entre a presença de cantinas $e$ a disponibilidade de alimentos ultraprocessados nas cantinas com o consumo destes alimentos, dentro da escola, entre adolescentes do 9o ano do Ensino Fundamental das redes pública e particular do Município de São Paulo, Brasil. Foi realizado um estudo transversal com dados do SP-Proso, em amostra de 2.680 adolescentes. Foram feitos modelos de regressão linear multinivel para avaliar as associações entre as exposições presença de cantinas nas escolas e disponibilidade de alimentos ultraprocessados com desfechos de frequência de consumo de ultraprocessados na escola. A presença de cantinas esteve associada à maior frequência de consumo de embutidos (0,46; IC95\%: 0,24; 0,68), salgadinhos de pacote (0,50; IC95\%: 0,19; 0,80), guloseimas (0,82; IC95\%: 0,55; 1,09) e bebidas açucaradas (0,34; IC95\%: 0,06; 0,62), bem como um escore de frequência de consumo de ultraprocessados (2,37; IC95\%: 1,25; 3,48). A disponibilidade de salgadinhos de pacote, guloseimas e bebidas açucaradas nas cantinas aumentou a frequência de consumo desses alimentos. Foi observado um efeito dose/resposta entre a diversidade de ultraprocessados nas cantinas e a frequência de consumo destes alimentos. Um ambiente alimentar escolar com maior disponibilidade de ultraprocessados está associado ao maior consumo dos mesmos na escola, o que aponta para a necessidade de regulamentar o comércio de alimentos dentro destas instituições.

Alimentação Escolar; Adolescente; Análise Multinível

Correspondência

M. A. Leite

Av. Dr. Arnaldo 455, São Paulo, SP 01246903, Brasil.

maria.alvim.leite@gmail.com

1 Universidade de São Paulo, São Paulo, Brasil.

2 Universidade Federal de Uberlândia, Uberlândia, Brasil.

3 Secretaria de Estado da Saúde de São Paulo, São Paulo, Brasil. 


\section{Introdução}

Alimentos ultraprocessados são formulações industriais tipicamente prontas para o consumo, produzidas com inúmeros ingredientes derivados de alimentos 1. Comparados a preparações culinárias e aos alimentos processados, os ultraprocessados têm, em média, maior teor de açúcar livre, gordura, sódio, calorias, aromatizantes, corantes e texturizantes, além de menor densidade de proteínas, fibras e micronutrientes $2,3,4$. São produtos que podem afetar o controle da saciedade e as respostas glicêmicas do organismo 5 , e seu consumo excessivo é um fator de risco para desfechos negativos em saúde de adolescentes, como síndrome metabólica 6 , excesso de peso 7 e asma e chiado 8 . Segundo os dados da Pesquisa Nacional de Saúde do Escolar (PeNSE), o consumo frequente de alimentos ultraprocessados entre adolescentes escolares brasileiros aumentou de 2009 a $2015^{9}$, quando atingiu 60,6\% 10 .

Uma vez que adolescentes permanecem por longos períodos nas escolas, consumindo ali entre um terço e metade das refeições diárias, reconhece-se que o ambiente alimentar escolar exerce um papel importante para a qualidade da sua alimentação 11,12. Segundo a Organização para Alimentação e Agricultura das Nações Unidas (FAO), o ambiente alimentar das escolas compreende espaços, infraestruturas e condições dentro e fora das instalações escolares onde os alimentos estão disponíveis, são obtidos ou comprados e consumidos. Envolve, também, as informações sobre alimentação e nutrição, e a promoção e a precificação de alimentos e produtos alimentícios (propagandas, marcas, rótulos de alimentos, embalagens, promoções etc.). $\mathrm{O}$ ambiente alimentar modifica o quão acessíveis, desejáveis e convenientes são os alimentos ali ofertados. Um ambiente alimentar escolar saudável permite e incentiva a comunidade escolar - crianças, adolescentes, famílias e funcionários da escola - a fazer escolhas que contribuam para melhores padrões alimentares 13 . Nesse sentido, estudos apontam que a existência de cantinas comerciais em escolas associa-se a um maior consumo de ultraprocessados por adolescentes 14,15,16,17,18, tornando-se necessária uma maior regulamentação dos alimentos disponíveis para venda e consumo no ambiente escolar.

Nas escolas públicas brasileiras, há o Programa Nacional de Alimentação Escolar (PNAE) e o Programa Saúde na Escola (PSE), que por meio do fornecimento gratuito de refeições e estratégias educativas protegem em certa medida a alimentação dos estudantes 19,20. Por outro lado, em 2015, 45,2\% dos alunos no Brasil estudavam em escolas com cantinas que comercializam alimentos $14 \mathrm{e}$, segundo dados do Estudo dos Riscos Cardiovasculares em Adolescentes (ERICA. http://www.erica.ufrj.br/) de 2013 e 2014, a venda de alimentos em escolas brasileiras acontecia em 45\% das públicas e 97,7\% das particulares ${ }^{21}$. No Brasil, a venda de alimentos nas cantinas comerciais pode ou não ser regulamentada, de acordo com cada estado, cidade, dependência administrativa e normas internas de cada escola 22.

Desconhecemos estudos brasileiros que avaliaram possíveis efeitos da presença de cantina e da qualidade dos alimentos vendidos nelas e o consumo de alimentos por adolescentes, especificamente durante o período das aulas. Tais explorações são necessárias para defender a expansão, fiscalização ou possíveis modificações nas leis brasileiras que regulamentam a venda de alimentos nas escolas, no intuito de melhorar as práticas alimentares, prevenir a obesidade e promover a saúde de adolescentes.

O objetivo deste estudo foi investigar a associação entre a presença de cantinas e a disponibilidade de alimentos ultraprocessados nas cantinas com o consumo destes alimentos, dentro da escola, entre adolescentes do 9o ano do Ensino Fundamental das redes pública e particular do Município de São Paulo, Brasil.

\section{Métodos}

O Projeto São Paulo para o Desenvolvimento Social de Crianças e Adolescentes (SP-Proso) é um estudo transversal realizado pelo Departamento de Medicina Preventiva, Faculdade de Medicina, Universidade de São Paulo. Conta com uma amostra representativa de adolescentes cursando o 9o ano do Ensino Fundamental II de escolas públicas e particulares do Município de São Paulo, capital do seu estado e maior cidade do Brasil, com aproximadamente 12 milhões de habitantes. No ano de 2017, havia 99.179 adolescentes cursando o 9 o ano 23 .

A amostra foi estratificada de acordo com o tipo de escola (pública municipal, pública estadual e particular) e o sorteio foi por conglomerados, considerando as turmas escolares como unidades pri- 
márias de amostragem. O tamanho da amostra foi estimado em 2.849 para permitir estimativas tão baixas quanto $15 \%$ com uma precisão de 0,06 e design effect $($ deff $)=1,724$. Dentre as 156 escolas sorteadas, 119 (87 públicas e 32 privadas) concordaram em participar. Foram selecionadas aleatoriamente uma turma de cada escola. Os adolescentes elegíveis foram aqueles que estavam presentes em sala de aula no dia da coleta de dados, cujos pais não se manifestaram contrários à sua participação e que não apresentassem comprometimento grave que limitasse a compreensão das questões ou a possibilidade de responder à pesquisa de forma anônima.

No total, 2.816 alunos estavam presentes na escola no dia da coleta de dados, dos quais 96 recusaram-se a participar e 18 foram excluídos por apresentarem deficiências cognitivas graves que comprometeram a sua capacidade de compreensão ou de responder ao questionário de forma anônima $(\mathrm{n}=17)$ ou problema disciplinar grave $(\mathrm{n}=1)$ que impediu a permanência em sala por decisão da gestão escolar. Outros 22 alunos foram excluídos por terem respondido menos de $20 \%$ do questionário, resultando numa amostra final de 2.680 alunos ( $94 \%$ da amostra estimada). Mais detalhes sobre este estudo podem ser lidos no relatório de Peres et al. 25.

Os dados foram coletados entre agosto e novembro de 2017, em horários de aulas, por meio de questionários de papel autopreenchidos. Em cada escola visitada, um auxiliar de pesquisa coletou dados acerca das características da escola e da cantina por meio de um instrumento de observação.

O questionário aplicado aos adolescentes continha perguntas sobre aspectos sociodemográficos e sobre alimentação. As perguntas sobre acesso a bens e serviços foram extraídas do questionário da PeNSE de 2012 26. As questões sobre a frequência de consumo de alimentos foram adaptadas do questionário da PeNSE de 2015 27. Os indicadores de práticas alimentares usados na PeNSE são validados 28 .

O instrumento de observação continha itens a respeito da presença de cantinas e um inventário de alimentos vendidos naquele espaço, adaptados do questionário da PeNSE de 2015 26. Mais detalhes sobre os instrumentos utilizados na pesquisa podem ser vistos no relatório do SP-Proso 25.

\section{Variáveis de interesse}

Como exposições, usamos as variáveis sobre a existência ou não de cantinas comerciais nas escolas e um escore da venda de alguns alimentos ultraprocessados, marcadores de padrões de consumo alimentar não saudável. Os subgrupos de alimentos cujo comércio foi usado para a construção desse escore foram biscoitos ou bolachas industrializadas, salgadinhos de pacote, guloseimas, refrigerantes e outras bebidas açucaradas. $\mathrm{O}$ escore poderia variar de 0 a 5 pontos, em que cada subgrupo disponível à venda no dia da coleta de dados correspondia a um ponto (por exemplo, biscoitos ou bolachas disponíveis à venda correspondiam a um ponto). O escore foi dividido em terços, de acordo com a sua distribuição na amostra, de forma que adolescentes do primeiro terço eram expostos a cantinas com menor disponibilidade de alimentos ultraprocessados e os do terceiro terço a cantinas com maior disponibilidade. Não foram consideradas variedades, marcas ou quantidades dos alimentos para a composição do escore.

Como desfecho, consideramos a frequência do consumo, dentro da escola, de alguns alimentos ultraprocessados. Foi questionada a frequência semanal, em dias letivos e dentro da escola ( 0 a 5 vezes), do consumo de cinco subgrupos de alimentos ultraprocessados: embutidos (hambúrguer, salsicha, mortadela, salame, presunto, nuggets ou linguiça), biscoitos ou bolachas (doces ou salgados), salgadinhos de pacote (incluindo batata frita de pacote), guloseimas (doces, balas, chocolates, chicletes, bombons ou pirulitos) e bebidas açucaradas (refrigerantes, sucos de pozinho, refrescos ou sucos de caixinha). A pergunta foi feita da seguinte forma: "Em uma semana de aulas comum (cinco dias letivos), em quantos dias você comeu ou bebeu na escola (inclua os alimentos que você comeu durante o intervalo, mesmo que seja do lado de fora da escola)?", seguida da lista de alimentos com as opções de frequência, de nenhum dia a cinco dias.

Em nossas análises, a frequência semanal do consumo desses subgrupos de alimentos foi somada para a construção de um segundo escore, em que cada dia de consumo de cada alimento correspondia a um ponto (por exemplo, consumir embutidos uma vez por semana correspondia a um ponto), podendo variar de 0 a 25 pontos. Os desfechos ora eram o consumo de cada subgrupo, isoladamente, ora o escore da soma das frequências de consumo. 
Como variáveis de confusão, incluímos as características individuais e das escolas. As variáveis individuais utilizadas foram: idade (em anos), sexo (masculino e feminino), escolaridade materna (até o Ensino Fundamental completo, Ensino Médio incompleto ou completo e Ensino Superior incompleto ou completo), cor da pele referida (branca, negra e outras) e situação socioeconômica. A situação socioeconômica das famílias dos adolescentes foi avaliada com base na posse de bens de consumo (telefone fixo, carro na família, computador e televisão no quarto e celular), ter banheiro em casa e o fato de ter uma empregada doméstica trabalhando em seus domicílios, perguntas extraídas do questionário da PeNSE de 2012 19. Com essas informações, foi calculado um escore contínuo, posteriormente categorizado em terços, de acordo com a metodologia proposta por Levy et al. 29. Quanto maior a prevalência desses itens, menor o seu peso na criação desse escore.

Como variáveis da escola, utilizamos: dependência administrativa (pública ou particular), tamanho da escola (de 200 a 500 estudantes, de 500 a 1.000 e com mais de 1.000 estudantes), turno das aulas (integral, manhã e tarde), ausência de refeitório estruturado, presença de ponto alternativo de venda de alimentos dentro da escola, presença de vendedor de alimentos ambulante em frente à escola no horário de término das aulas, presença de propaganda de alimentos ultraprocessados dentro da escola (pelo menos uma propaganda dos seguintes subgrupos de alimentos: biscoitos ou bolachas industrializados, salgadinhos de pacote, guloseimas, refrigerantes e outras bebidas açucaradas).

\section{Análises dos dados}

Inicialmente, médias e prevalências das características dos adolescentes e das escolas foram descritas para o total da amostra e segundo a condição de estudar em escolas sem ou com cantina.

Em seguida, fizemos modelos de regressão linear multinível, considerando como níveis: (i) o indivíduo e (ii) as escolas, para avaliar a relação entre a presença de cantina na escola e a frequência de consumo dos subgrupos de alimentos ultraprocessados analisados. Fizemos também modelos multiníveis para testar a associação entre a venda de cada subgrupo de alimentos na cantina e a sua frequência de consumo. Depois, testamos a associação entre a presença de cantinas e o escore da frequência de consumo de ultraprocessados. E, por fim, fizemos regressões lineares multiníveis para testar a relação de dose/resposta entre a quantidade de ultraprocessados vendidos na cantina e o escore de frequência de consumo destes alimentos.

A análise multinível permite lidar simultaneamente com as relações entre exposição, desfecho e covariáveis, tanto no nível do indivíduo quanto no nível do grupo ou do contexto ao qual pertencem. Dessa forma, a dependência dos sujeitos em relação ao grupo em que estão inseridos foi considerada 30 .

Os modelos multiníveis foram feitos em dois passos, além de um primeiro modelo bruto. Nos modelos ajustados, primeiro foram inseridas as variáveis sociodemográficas individuais, a dependência administrativa da escola, o tamanho da escola e o turno das aulas. Posteriormente, foram incluídas, além dessas variáveis de ajustes, outras variáveis de características do ambiente alimentar escolar (ausência de refeitório estruturado, presença de ponto alternativo de venda de alimentos, presença de vendedor ambulante na porta da escola e presença de propaganda de alimentos ultraprocessados).

A imputação múltipla por equações encadeadas foi usada para atribuir valores numéricos ao nível educacional da mãe, que possuía $25,8 \%$ de valores ausentes, conforme metodologia descrita por outros estudos 15,31. Os dados imputados exibiram reprodutibilidade estatística satisfatória, de acordo com a análise de erro de Monte Carlo 32.

As análises foram ponderadas de acordo com o desenho amostral e foram feitas utilizando-se o Stata versão 14.0 (https://www.stata.com/).

\section{Aspectos éticos}

De acordo com a Declaração de Helsinki, o projeto foi aprovado pelo Comitê de Ética em Pesquisa da Faculdade de Medicina, Universidade de São Paulo (CEP-FMUSP; parecer no 1.719.856) e pela Comissão Nacional de Ética em Pesquisa (CONEP), ligada ao Conselho Nacional de Saúde (CNS) (parecer no 2.014.816). Apenas os adolescentes cujos pais não se opuseram à participação na pesquisa foram convidados a responder ao questionário anônimo. Um Termo de Assentimento Livre e Esclarecido foi apresentado aos adolescentes. 


\section{Resultados}

As características dos 2.680 adolescentes e das 119 escolas, de acordo com a presença ou não de cantina, são apresentadas na Tabela 1. A maior parte dos estudantes era do sexo masculino, independentemente da presença ou não de cantina (52,32\% e 52,67\%, respectivamente); os alunos de escolas sem cantinas eram mais velhos (15,0 anos versus 14,8 anos) e a maioria se declarou negra $(59,1 \%)$; já entre os alunos das escolas com cantina, houve a mesma proporção de brancos e negros $(46,8 \%$ e $46,3 \%$, respectivamente) e observamos maior escolaridade materna e melhor condição socioeconômica quando comparados aos alunos de escolas sem cantina. Não havia nenhum estudante de instituição particular em escola sem cantina (Tabela 1$)$.

\section{Tabela 1}

Características de estudantes e escolas de acordo com a existência de cantinas. SP-Proso, 2017 (N = 2.680).

\begin{tabular}{|c|c|c|c|c|c|c|}
\hline & \multicolumn{2}{|c|}{ Total } & \multicolumn{2}{|c|}{$\begin{array}{c}\text { Estudantes de escolas sem } \\
\text { cantinas }(20,4 \%)\end{array}$} & \multicolumn{2}{|c|}{$\begin{array}{c}\text { Estudantes de escolas com } \\
\text { cantinas }(79,6 \%)\end{array}$} \\
\hline & $\%$ & IC95\% & $\%$ & IC95\% & $\%$ & IC95\% \\
\hline \multicolumn{7}{|l|}{ Características individuais } \\
\hline Idade (anos) [média] & 14,8 & 14,$8 ; 14,9$ & 15,0 & 15,$0 ; 15,1$ & 14,8 & 14,$7 ; 14,8$ \\
\hline \multicolumn{7}{|l|}{ Sexo } \\
\hline Feminino & 47,4 & 45,$7 ; 49,1$ & 47,7 & 43,$4 ; 52,0$ & 47,3 & 45,$5 ; 49,1$ \\
\hline Masculino & 52,6 & 50,$9 ; 54,3$ & 52,3 & 48,$0 ; 56,6$ & 52,7 & 50,$8 ; 54,5$ \\
\hline \multicolumn{7}{|l|}{ Escolaridade materna } \\
\hline Até o Ensino Fundamental completo & 24,8 & 22,$7 ; 27,0$ & 34,2 & 31,$2 ; 37,4$ & 22,4 & 19,$8 ; 25,2$ \\
\hline Ensino Médio incompleto ou completo & 40,2 & 37,$5 ; 42,9$ & 44,1 & 40,$0 ; 48,2$ & 39,2 & 35,$9 ; 42,5$ \\
\hline Ensino Superior incompleto ou completo & 35,0 & 32,$1 ; 38,1$ & 21,7 & 17,$9 ; 26,0$ & 38,4 & 34,$5 ; 42,5$ \\
\hline \multicolumn{7}{|l|}{ Escore de bens e serviços } \\
\hline Baixo & 33,5 & 30,$6 ; 36,6$ & 43,9 & 38,$2 ; 49,7$ & 31,0 & 27,$6 ; 34,6$ \\
\hline Médio & 40,0 & 37,$0 ; 43,0$ & 39,5 & 34,$2 ; 45,1$ & 40,1 & 36,$6 ; 43,7$ \\
\hline Alto & 26,5 & 23,$4 ; 29,9$ & 16,6 & 13,$8 ; 19,8$ & 28,9 & 25,$1 ; 33,1$ \\
\hline \multicolumn{7}{|l|}{ Cor da pele } \\
\hline Branca & 44,2 & 41,$7 ; 46,8$ & 34,0 & 31,$1 ; 37,0$ & 46,8 & 43,$6 ; 50,2$ \\
\hline Negra & 49,1 & 46,$3 ; 51,9$ & 59,6 & 55,$0 ; 64,1$ & 46,3 & 42,$7 ; 49,9$ \\
\hline Outras & 6,7 & 5,$5 ; 8,1$ & 6,3 & 4,$0 ; 10,0$ & 6,8 & 5,$5 ; 8,4$ \\
\hline \multicolumn{7}{|l|}{$\begin{array}{l}\text { Frequência média de consumo de alimentos } \\
\text { ultraprocessados na escola (dias) [mínimo = } 0 \\
\text { máximo }=5 \text { ) }\end{array}$} \\
\hline Embutidos & 1,3 & 1,$2 ; 1,4$ & 1,0 & 0,$9 ; 1,2$ & 1,3 & 1,$2 ; 1,4$ \\
\hline Biscoitos ou bolachas & 2,2 & 2,$1 ; 2,3$ & 2,1 & 1,$9 ; 2,3$ & 2,2 & 2,$1 ; 2,0$ \\
\hline Salgadinhos de pacote & 1,5 & 1,$4 ; 1,6$ & 1,4 & 1,$2 ; 1,5$ & 1,6 & 1,$4 ; 1,7$ \\
\hline Guloseimas & 2,2 & 2,$1 ; 2,3$ & 1,8 & 1,$7 ; 2,0$ & 2,3 & 2,$2 ; 2,5$ \\
\hline Bebidas açucaradas & 1,9 & 1,$8 ; 2,0$ & 1,6 & 1,$4 ; 1,8$ & 1,9 & 1,$8 ; 2,1$ \\
\hline $\begin{array}{l}\text { Escore de consumo de ultraprocessados na } \\
\text { escola }[\text { mínimo }=0 ; \text { máximo }=25]\end{array}$ & 9,1 & 8,$6 ; 9,5$ & 7,8 & 7,$1 ; 8,6$ & 9,4 & 8,$9 ; 9,8$ \\
\hline
\end{tabular}

(continua) 


\begin{tabular}{|c|c|c|c|c|c|c|}
\hline & \multicolumn{2}{|c|}{ Total } & \multicolumn{2}{|c|}{$\begin{array}{c}\text { Estudantes de escolas sem } \\
\text { cantinas }(20,4 \%)\end{array}$} & \multicolumn{2}{|c|}{$\begin{array}{l}\text { Estudantes de escolas com } \\
\text { cantinas }(79,6 \%)\end{array}$} \\
\hline & $\%$ & IC95\% & $\%$ & IC95\% & $\%$ & IC95\% \\
\hline \multicolumn{7}{|l|}{ Características da escola } \\
\hline \multicolumn{7}{|l|}{ Dependência administrativa da escola } \\
\hline Particular & 27,1 & 27,$1 ; 27,1$ & 0,0 & - & 100,0 & - \\
\hline Pública & 72,9 & 72,$9 ; 72,9$ & 36,1 & 33,$0 ; 39,3$ & 63,9 & 60,$7 ; 67,0$ \\
\hline \multicolumn{7}{|l|}{ Tamanho da escola (estudantes) } \\
\hline $200-500$ & 9,9 & 5,$1 ; 18,5$ & 8,8 & 2,$1 ; 29,8$ & 10,3 & 4,$8 ; 20,8$ \\
\hline $500-1.000$ & 47,7 & 36,$7 ; 57,7$ & 59,7 & 41,$7 ; 75,4$ & 42,6 & 30,$4 ; 55,9$ \\
\hline Mais de 1.000 & 43,0 & 32,$8 ; 53,9$ & 31,5 & 17,$6 ; 49,6$ & 47,0 & 34,$4 ; 60,1$ \\
\hline \multicolumn{7}{|l|}{ Turno das aulas } \\
\hline Integral & 1,5 & 0,$2 ; 9,9$ & 0 & - & 1,9 & 0,$3 ; 12,9$ \\
\hline Manhã & 70,2 & 59,$6 ; 78,9$ & 70,7 & 52,$8 ; 83,9$ & 70,0 & 57,$0 ; 80,4$ \\
\hline Tarde & 28,4 & 19,$8 ; 38,9$ & 29,3 & 16,$1 ; 47,2$ & 28,1 & 18,$0 ; 41,0$ \\
\hline \multicolumn{7}{|l|}{$\begin{array}{l}\text { Escore médio de ultraprocessados vendidos } \\
\text { nas cantinas }[\text { mínimo }=0 ; \text { máximo }=5] \text { * }\end{array}$} \\
\hline Primeira categoria (escore médio $=2,30$ ) & - & - & - & - & 23,8 & 15,$9 ; 34,2$ \\
\hline Segunda categoria (escore médio $=4,00$ ) & - & - & - & - & 28,6 & 18,$4 ; 41,5$ \\
\hline Terceira categoria (escore médio $=5,00$ ) & - & - & - & - & 47,6 & 35,$1 ; 60,4$ \\
\hline Ausência de refeitório estruturado & 14,7 & 8,$5 ; 24,3$ & 13,7 & 4,$4 ; 35,1$ & 15,0 & 7,$9 ; 26,5$ \\
\hline $\begin{array}{l}\text { Presença de ponto alternativo de venda de } \\
\text { alimentos }\end{array}$ & 3,7 & 1,$5 ; 8,8$ & 1,4 & 0,$2 ; 9,5$ & 4,5 & 1,$7 ; 11,3$ \\
\hline $\begin{array}{l}\text { Presença de vendedor ambulante em frente à } \\
\text { escola no horário de término das aulas }\end{array}$ & 22,3 & 15,$3 ; 31,4$ & 23,4 & 12,$5 ; 39,6$ & 21,9 & 13,$6 ; 33,3$ \\
\hline $\begin{array}{l}\text { Presença de propaganda de alimentos } \\
\text { ultraprocessados ** }\end{array}$ & 5,5 & 2,$4 ; 11,9$ & 2,7 & 0,$6 ; 10,6$ & 6,4 & $(2,6 ; 15,1)$ \\
\hline
\end{tabular}

IC95\%: intervalo de 95\% de confiança.

* Alimentos ultraprocessados incluídos no escore: biscoitos ou bolachas industrializados, salgadinhos de pacote, guloseimas, refrigerantes e outras bebidas açucaradas;

** Pelo menos uma propaganda dos seguintes alimentos: biscoitos ou bolachas industrializados, salgadinhos de pacote, guloseimas, refrigerantes e outras bebidas açucaradas.

Sobre as características do ambiente alimentar escolar, observamos que 14,7\% deles não tinham refeitório estruturado, $3,7 \%$ das escolas tinham ponto alternativo de venda de alimentos; $22,3 \%$ contavam com vendedores ambulantes na porta; e 5,5\% tinham propaganda de alimentos. Não houve diferenças, para essas características, entre escolas com e sem cantinas.

$\mathrm{Na}$ Tabela 2, observamos associações entre a presença de cantinas e a frequência de consumo de alimentos ultraprocessados na escola. No modelo 1, que não incluía as covariáveis do ambiente alimentar escolar além da presença de cantina, observamos que ter cantina gerou um aumento médio de 0,52 (IC95\%: 0,30; 0,74) na frequência semanal de consumo, na escola, de embutidos; de 0,31 (IC95\%: 0,07; 0,55) para biscoitos ou bolachas; de 0,59 (IC95\%: 0,33; 0,86) para salgadinhos de pacote; de 0,82 (IC95\%: 0,58; 1,06) para guloseimas; e de 0,39 (IC95\%: 0,14; 0,64) para bebidas açucaradas. Após o ajuste para as demais covariáveis (modelo 2), os coeficientes apresentaram pequenas reduções e o consumo de biscoitos e bolachas perdeu significância estatística. Assim, houve um aumento médio de 0,46 (IC95\%: 0,24; 0,68) para embutidos, 0,50 (IC95\%: 0,19; 0,80) para salgadinhos de pacote, 0,82 (IC95\%: 0,55; 1,09) para guloseimas e 0,34 (IC95\%: 0,06; 0,62) para bebidas açucaradas (Tabela 2).

Quando analisamos se a disponibilidade de cada alimento, na cantina, estava associada à maior frequência de consumo do mesmo na escola, observamos associações significativas para todos os alimentos, exceto para biscoitos ou bolachas (Tabela 3). Nos modelos 1 e 2, a venda de salgadinhos de pacote aumentou a frequência semanal média de consumo deste alimento na escola em 0,45 (IC95\%: 0,23; 0,67) e 0,38 (IC95\%: 0,13; 0,63), respectivamente; para guloseimas, os coeficientes de aumento 
Tabela 2

Coeficientes e intervalos de 95\% de confiança (IC95\%) para a frequência de consumo de alimentos ultraprocessados na escola, de acordo com a presença de cantina. SP-Proso, 2017 ( $\mathrm{N}=2.680)$.

\begin{tabular}{|c|c|c|c|c|c|c|c|c|c|}
\hline \multirow{2}{*}{$\begin{array}{l}\text { Frequência } \\
\text { de consumo } \\
\text { de alimentos } \\
\text { ultraprocessados } \\
\text { na escola }\end{array}$} & \multicolumn{3}{|c|}{ Modelos brutos } & \multicolumn{3}{|c|}{ Modelos ajustados 1 * } & \multicolumn{3}{|c|}{ Modelos ajustados 2 ** } \\
\hline & Coeficiente & IC95\% & Valor de $p$ & Coeficiente & IC95\% & Valor de $p$ & Coeficiente & IC95\% & Valor de $p$ \\
\hline Embutidos & 0,28 & 0,$11 ; 0,46$ & 0,002 & 0,52 & 0,$30 ; 0,74$ & $<0,001$ & 0,46 & 0,$24 ; 0,68$ & $<0,001$ \\
\hline $\begin{array}{l}\text { Biscoitos ou } \\
\text { bolachas }\end{array}$ & 0,15 & $-0,05 ; 0,35$ & 0,149 & 0,31 & 0,$07 ; 0,55$ & 0,012 & 0,21 & $-0,06 ; 0,48$ & 0,135 \\
\hline $\begin{array}{l}\text { Salgadinhos de } \\
\text { pacote }\end{array}$ & 0,23 & 0,$01 ; 0,45$ & 0,037 & 0,59 & 0,$33 ; 0,86$ & $<0,001$ & 0,50 & 0,$19 ; 0,80$ & 0,001 \\
\hline Guloseimas & 0,49 & 0,$30 ; 0,69$ & $<0,001$ & 0,82 & 0,$58 ; 1,06$ & $<0,001$ & 0,82 & 0,$55 ; 1,09$ & $<0,001$ \\
\hline Bebidas açucaradas & 0,36 & 0,$16 ; 0,57$ & 0,001 & 0,39 & 0,$14 ; 0,64$ & 0,003 & 0,34 & 0,$06 ; 0,62$ & 0,017 \\
\hline
\end{tabular}

Nota: Referência = ausência de cantinas.

* Modelos 1 = modelos brutos + idade, sexo, escolaridade materna, escore de bens e serviços, cor da pele, dependência administrativa da escola, tamanho da escola e turno das aulas;

** Modelos 2 = modelos 1 + ausência de refeitório estruturado, presença de ponto alternativo de venda de alimentos, presença de vendedor ambulante em frente à escola no horário de término das aulas e presença de propaganda de alimentos ultraprocessados.

Tabela 3

Coeficientes e intervalos de 95\% de confiança (IC95\%) para a frequência de consumo de alimentos ultraprocessados na escola, de acordo com a disponibilidade destes alimentos na cantina. SP-Proso, $2017(\mathrm{~N}=2.680)$.

\begin{tabular}{|c|c|c|c|c|c|c|c|c|c|}
\hline \multirow{2}{*}{$\begin{array}{l}\text { Frequência } \\
\text { de consumo } \\
\text { de alimentos } \\
\text { ultraprocessados na } \\
\text { escola }\end{array}$} & \multicolumn{3}{|c|}{ Modelos brutos } & \multicolumn{3}{|c|}{ Modelos ajustados 1 * } & \multicolumn{3}{|c|}{ Modelos ajustados 2 ** } \\
\hline & Coeficiente & IC95\% & Valor de $p$ & Coeficiente & IC95\% & Valor de $p$ & Coeficiente & IC95\% & Valor de p \\
\hline Biscoitos ou bolachas & 0,06 & $-0,14 ; 0,26$ & 0,556 & 0,09 & $-0,12 ; 0,31$ & 0,386 & 0,08 & $-0,13 ; 0,30$ & 0,441 \\
\hline Salgadinhos de pacote & 0,35 & 0,$14 ; 0,56$ & 0,001 & 0,45 & 0,$23 ; 0,67$ & $<0,001$ & 0,38 & 0,$13 ; 0,63$ & 0,002 \\
\hline Guloseimas & 0,51 & 0,$32 ; 0,70$ & $<0,001$ & 0,69 & 0,$47 ; 0,91$ & $<0,001$ & 0,69 & 0,$44 ; 0,94$ & $<0,001$ \\
\hline Bebidas açucaradas & 0,37 & 0,$16 ; 0,57$ & $<0,001$ & 0,38 & 0,$15 ; 0,61$ & 0,001 & 0,35 & 0,$10 ; 0,59$ & 0,005 \\
\hline
\end{tabular}

Nota: Referência = ausência de cantina ou ausência do alimento na cantina.

* Modelos 1 = modelos brutos + idade, sexo, escolaridade materna, escore de bens e serviços, cor da pele, dependência administrativa da escola, tamanho da escola e turno das aulas.;

** Modelos 2 = modelos 1 + ausência de refeitório estruturado, presença de ponto alternativo de venda de alimentos, presença de vendedor ambulante em frente à escola no horário de término das aulas e presença de propaganda de alimentos ultraprocessados.

foram de 0,69 para os dois modelos (no modelo 1: IC95\%: 0,55; 1,09 e no modelo 2: IC95\%: 0,44; 0,94); para bebidas açucaradas, os coeficientes de aumento foram de 0,38 (modelo 1: IC95\%: 0,55; 1,09) e 0,35 (modelo 2: IC95\%: 0,55; 1,09) (Tabela 3).

A presença de cantinas nas escolas se associou a um aumento na média do escore de frequência de consumo de ultraprocessados em 2,37 (IC95\%: 1,25; 3,48) no modelo 2. Além disso, observamos um gradiente na associação, em que quanto maior a disponibilidade de alimentos ultraprocessados em cantinas, maior o escore de frequência de consumo destes alimentos, independentemente do ajuste realizado (Tabela 4 ). 
Tabela 4

Coeficientes e intervalos de 95\% de confiança (IC95\%) para o escore de frequência de consumo de alimentos ultraprocessados na escola, de acordo com a presença de cantina e com o escore de ultraprocessados vendidos na cantina. SP-Proso, 2017 ( $N=2.680)$.

\begin{tabular}{|c|c|c|c|c|c|c|c|c|c|}
\hline & \multicolumn{3}{|c|}{ Modelos brutos } & \multicolumn{3}{|c|}{ Modelos ajustados 1 * } & \multicolumn{3}{|c|}{ Modelos ajustados 2 ** } \\
\hline & Coeficiente & IC95\% & Valor de $p$ & Coeficiente & IC95\% & Valor de $p$ & Coeficiente & IC95\% & Valor de $p$ \\
\hline $\begin{array}{l}\text { Exposição: presença } \\
\text { de cantinas }\end{array}$ & 1,50 & 0,$69 ; 2,32$ & $<0,001$ & 2,61 & 1,$60 ; 3,61$ & $<0,001$ & 2,37 & 1,$25 ; 3,48$ & $<0,001$ \\
\hline $\begin{array}{l}\text { Exposição: escore } \\
\text { de ultraprocessados } \\
\text { vendidos nas } \\
\text { cantinas (mínimo = } \\
0 ; \text { máximo }=5 \text { ) } \star \star \star\end{array}$ & & & & & & & & & \\
\hline $\begin{array}{l}\text { Primeira categoria } \\
(\text { escore médio = } \\
2,30)\end{array}$ & 0,21 & $-0,90 ; 1,32$ & $<0,001$ & 1,16 & $-0,35 ; 2,68$ & $<0,001$ & 1,11 & $-0,39 ; 2,61$ & $0,011 \#$ \\
\hline $\begin{array}{l}\text { Segunda } \\
\text { categoria (escore } \\
\text { médio }=4,00 \text { ) }\end{array}$ & 1,68 & 0,$65 ; 2,72$ & $<0,001$ & 2,37 & 1,$24 ; 3,50$ & $<0,001$ & 2,18 & 0,$94 ; 3,41$ & $0,011 \#$ \\
\hline $\begin{array}{l}\text { Terceira categoria } \\
(\text { escore médio = } \\
5,00)\end{array}$ & 2,23 & 1,$30 ; 3,15$ & $<0,001$ & 2,90 & 1,$79 ; 4,02$ & $<0,001$ & 2,72 & 1,$47 ; 3,98$ & $0,011 \#$ \\
\hline
\end{tabular}

Nota: referência = ausência de cantinas.

* Modelos 1 = modelos brutos + idade, sexo, escolaridade materna, escore de bens e serviços, cor da pele, dependência administrativa da escola, tamanho da escola e turno das aulas;

** Modelos 2 = modelos 1 + ausência de refeitório estruturado, presença de ponto alternativo de venda de alimentos, presença de vendedor ambulante em frente à escola no horário de término das aulas e presença de propaganda de alimentos ultraprocessados;

*** Alimentos ultraprocessados incluídos no escore: biscoitos ou bolachas industrializados, salgadinhos de pacote, guloseimas, refrigerantes e outras bebidas açucaradas;

\# Valor de p de tendência.

As covariáveis de ambiente alimentar ausência de refeitório estruturado, presença de ponto alternativo de venda de alimentos, presença de vendedor ambulante na porta da escola e presença de propaganda de alimentos ultraprocessados não se associaram aos nossos desfechos (dados não apresentados).

\section{Discussão}

Neste estudo, realizado com uma amostra representativa de estudantes em uma das maiores cidades da América Latina, observamos que a presença de cantina nas escolas aumenta a frequência de consumo de alimentos ultraprocessados, medidos por um escore e por cada subgrupo de alimentos avaliado individualmente (embutidos, salgadinhos de pacote, guloseimas e bebidas açucaradas), exceto para o consumo de biscoitos ou bolachas. Observamos, também, que a venda de cada subgrupo nas cantinas aumenta o seu consumo, exceto para biscoitos ou bolachas, e que quanto maior a variedade de ultraprocessados disponíveis à venda nas cantinas, maior a frequência de consumo destes alimentos pelos adolescentes durante o período das aulas.

Outros estudos já mostraram achados que vão na mesma direção que os nossos. Azeredo et al.15, com dados da PeNSE de 2012, encontraram que a presença de cantinas que vendiam alimentos não saudáveis em escolas brasileiras aumentava o consumo de refrigerantes e salgados fritos pelos estu- 
dantes. Um estudo feito com dados da PeNSE de 2015 encontrou que a presença de cantinas comerciais nas escolas foi associada a uma maior probabilidade de consumir alimentos ultraprocessados salgados 14. Em Alberta e Ontario, no Canada, comprar o lanche na escola foi associado a um maior consumo de bebidas açucaradas 33. Outros estudos também encontraram relação entre comprar o lanche em cantinas e maior consumo de alimentos considerados não saudáveis 16,17,18.

Quanto maior a oferta de determinados produtos, maior será a sua demanda. Foi isso que nossos dados mostraram ao analisarmos o efeito dose/resposta entre a comercialização e o consumo de alimentos ultraprocessados. Em concordância com nossos achados, um estudo recente observou um aumento de 1,67\% no número de alimentos não saudáveis comprados por estudantes australianos nas escolas, para cada aumento de $1 \%$ na disponibilidade destes alimentos nas cantinas ${ }^{34}$. Essas relações ajudam a entender melhor como o ambiente pode influenciar nas escolhas alimentares dos adolescentes. Observa-se o papel do ambiente alimentar na modulação dos hábitos de consumo dos adolescentes, em que uma maior disponibilidade de produtos ultraprocessados será impulsionadora do seu consumo.

Os únicos alimentos que não tiveram associação, tanto com a presença de cantinas quanto com a venda, foram os biscoitos ou bolachas. É possível que biscoitos ou bolachas sejam adquiridos em outros locais, como supermercados e mercearias, e que sejam levados de casa para a escola. Isso explicaria por que o hábito de consumo desses alimentos foi menos relacionado à presença da cantina e sua disponibilidade à venda em relação aos demais alimentos estudados.

Nenhuma das outras covariáveis de ambiente alimentar (ausência de refeitório estruturado, presença de ponto alternativo de venda de alimentos, presença de vendedor ambulante na porta da escola e presença de propaganda de alimentos ultraprocessados) esteve associada aos nossos desfechos, mas foram mantidas nos modelos por permitirem o ajuste das associações das exposições principais. A baixa prevalência dessas variáveis nas escolas avaliadas pode justificar, em parte, a falta de associações. Alternativamente, elas podem realmente ser de menor importância quando comparadas ao papel exercido pela presença da cantina e pelos alimentos ali comercializados, como moduladores do consumo de alimentos ultraprocessados.

O ambiente escolar é considerado uma plataforma em que mudanças positivas nos comportamentos podem ser implementadas, visto que as intervenções podem ser facilmente entregues e avaliadas, uma vez que as escolas mantêm contato contínuo, direto e intenso com os estudantes, onde é possível criar um ambiente saudável e favorável a práticas educativas ${ }^{35}$. Modificações no ambiente alimentar escolar podem produzir importantes efeitos nos comportamentos alimentares e na saúde de estudantes ao permitir que façam escolhas mais saudáveis 12 , sendo mais efetivas que intervenções pontuais e/ou individuais de conscientização.

Um estudo, também realizado com dados da PeNSE de 2015, ao estimar quanto da variação individual na prevalência de comportamentos de saúde é atribuível ao nível escolar, mostrou a importância do contexto escolar na saúde do adolescente e destacou que as intervenções no ambiente escolar podem ser eficazes para promover comportamentos saudáveis 36 . Logo, parece plausível afirmar que regulamentar e restringir o que é vendido nas escolas pode melhorar a alimentação dos adolescentes.

Na cidade de São Paulo, já existem instrumentos legais que têm como objetivo promover a alimentação saudável de crianças e adolescentes escolares. Além dos programas nacionais PNAE e PSE, no Estado de São Paulo, há a Portaria Conjunta COGSP/CEI/DSE, de 23 de março de 2005, que dispõe sobre quais alimentos podem ser comercializados nas cantinas das escolas públicas estaduais. Esse, porém, não é um instrumento de regulamentação mandatória 37. Já no Município de São Paulo, há a Portaria no 11/2001, de 15 de fevereiro de 2001, que proíbe a existência de cantinas em todas as escolas da rede pública municipal 38 , o que representa um avanço, visto que não há uma regulamentação nacional acerca dos alimentos vendidos nas escolas, mas sim algumas leis espalhadas entre cidades brasileiras 22 . As escolas particulares do município, por outro lado, não são cobertas por nenhum tipo de regulamentação ou política no que diz respeito aos alimentos comercializados nestes espaços.

Em 2012, o Fundo Nacional de Desenvolvimento da Educação (FNDE) demonstrou um esforço em promover a venda de alimentos mais saudáveis em cantinas, por meio de uma nota técnica 39 . Esse, porém, é um instrumento orientador, também não mandatório, o que pode explicar uma provável baixa adesão. 
Modificar o ambiente alimentar por meio da restrição da venda de alguns alimentos em ambientes institucionais tem como vantagem ser uma medida de caráter universal, visto que todos os alunos serão afetados por aquela medida, diferentemente de outras estratégias motivacionais ou educacionais, que podem atingir os indivíduos de maneiras diferentes. Instrumentos legais são a ferramenta possível de ser aplicada em relação à venda de alimentos em cantinas escolares. Mas apenas a criação de leis locais não parece ser eficaz. Um estudo, que avaliou se a presença de leis que proibiam a venda de refrigerantes em escolas de capitais brasileiras diminuiriam a sua disponibilidade nesses espaços, encontrou que nem sempre a existência da regulamentação é suficiente para restringir a venda, apontando para a necessidade da existência de uma lei clara, única e nacional, e que seja acompanhada de fiscalização do seu cumprimento e avaliação constante 22.

O estudo, por ter um desenho transversal, implica que as medidas de efeito dele extraídas meçam associações e não relações causais. Apesar dessa possível limitação, acreditados que seja pouco provável, no caso deste estudo, que haja causalidade reversa. É plausível que a disponibilidade dos alimentos seja pregressa e determinante para as atitudes de compra dos adolescentes. Além disso, as medidas de consumo foram avaliadas por um indicador não validado e esta é uma limitação. Porém, de modo geral, questionários de frequência de consumo alimentar autorreferida são a forma mais comum de coleta de dados em inquéritos populacionais, o que nos garante comparabilidade com a literatura.

Como aspectos positivos do estudo, ressaltamos a avaliação do consumo somente dentro da escola (em até cinco dias da semana), que permite entender melhor como o ambiente alimentar escolar se associa com as métricas de consumo neste mesmo espaço, diferentemente de outros estudos feitos com dados da PeNSE 14,15 que avaliaram o consumo total (dentro e fora da escola, em sete dias da semana). Além disso, os dados das cantinas foram coletados em campo por pesquisadores treinados, em vez do uso de dados relatados por diretores de escolas, como utilizado em outros estudos 15,22 , que poderiam em alguma medida ser influenciados pelo viés de desejabilidade social. A validade externa do estudo é outro ponto positivo, visto que este apresentou alta taxa de resposta em uma amostra representativa de alunos de 9 o ano e, de acordo com as projeções feitas com base em dados da Pesquisa Nacional por Amostra de Domicílios Contínua (PNAD), 97,5\% das crianças e adolescentes, com idade compatível, estariam matriculadas no Ensino Fundamental na cidade de São Paulo em 201740.

O ineditismo deste estudo também é relevante. Nosso trabalho foi o primeiro a avaliar associações diretas entre a presença de cantina no consumo de alimentos ultraprocessados dentro da escola e o efeito dose/resposta entre a comercialização de ultraprocessados nas cantinas e o seu consumo no Brasil. Acreditamos que esses achados sejam de grande utilidade no delineamento de políticas públicas que visem a regulamentar a venda de alimentos nas escolas.

\section{Conclusão}

Nosso estudo demonstrou que a disponibilidade de alimentos não saudáveis nas escolas está associada de maneira significativa ao consumo alimentar dos adolescentes nestes ambientes. Compreendendo a escola como um espaço de proteção e educação, é importante que os alimentos ali oferecidos sejam promotores de padrões alimentares saudáveis e de saúde.

As evidências que encontramos apontam para a necessidade de regulamentar o comércio de alimentos dentro das escolas, seja por meio da proibição da existência de cantinas ou da regulamentação dos tipos de alimentos comercializados - sem perder de vista a importância da coexistência de programas e políticas que ofertem ou facilitem o acesso a alimentos frescos e saudáveis. 


\section{Colaboradores}

M. A. Leite contribuiu na concepção e execução da pesquisa, análise dos dados e redação do artigo. C. M. Azeredo colaborou na concepção e execução da pesquisa, análise dos dados e redação do artigo. M. F. T. Peres participou da concepção e execução da pesquisa, análise dos dados e redação do artigo. M. M. L. Escuder contribuiu no processo de elaboração da pesquisa, desenho do estudo, cálculo da amostra, análise dos dados e revisão da versão final do artigo. R. B. Levy colaborou na concepção e execução da pesquisa, análise dos dados e redação do artigo.

\section{Informações adicionais}

ORCID: Maria Alvim Leite (0000-0002-27747126); Catarina Machado Azeredo (0000-00026189-4429); Maria Fernanda Tourinho Peres (0000-0002-7049-905X); Maria Mercedes Loureiro Escuder (0000-0002-3103-2617); Renata Bertazzi Levy (0000-0002-6189-4429).

\section{Referências}

1. Monteiro CA, Cannon G, Levy RB, Moubarac JC, Louzada MLC, Rauber F, et al. Ultra-processed foods: what they are and how to identify them. Public Health Nutr 2019; 22:936-41.

2. Louzada MLDC, Martins APB, Canella DS, Baraldi LG, Levy RB, Claro RM, et al. Ultraprocessed foods and the nutritional dietary profile in Brazil. Rev Saúde Pública 2015; 49:38.

3. Louzada MLDC, Martins APB, Canella DS, Baraldi LG, Levy RB, Claro RM, et al. Impacto de alimentos ultraprocessados sobre o teor de micronutrientes da dieta no Brasil. Rev Saúde Pública 2015; 49:45.

4. Barcelos GT, Rauber F, Vitolo MR. Produtos processados e ultraprocessados e ingestão de nutrientes em crianças. Ciênc Saúde Colet 2014; 7:155-61.

5. Fardet A. Minimally processed foods are more satiating and less hyperglycemic than ultraprocessed foods: a preliminary study with 98 ready-to-eat foods. Food Funct 2016; 7:233846.

6. Tavares LF, Fonseca SC, Rosa MLG, Yokoo EM. Relationship between ultra-processed foods and metabolic syndrome in adolescents from a Brazilian Family Doctor Program. Public Health Nutr 2012; 15:82-7.

\section{Agradecimentos}

À Academia Britânica/Fundo Newton, à Fundação de Amparo à Pesquisa do Estado de São Paulo (FAPESP), ao Conselho Nacional de Desenvolvimento Científico e Tecnológico (CNPq) e à Coordenação de Aperfeiçoamento de Pessoal de Nível Superior (CAPES) pelo financiamento. O Projeto São Paulo para o Desenvolvimento Social de Crianças e Adolescentes (SP-Proso) conta com um financiamento da Academia Britânica, no âmbito do programa Newton Advanced Fellowship (R1/2016). Esse projeto conta, também, com financiamento já aprovado pela FAPESP (processo 2016/22259-4). Já o presente estudo, em separado, obteve um financiamento aprovado junto ao Conselho Nacional de Desenvolvimento Científico e Tecnológico (CNPq; processo 408360/2017). A autora M. A. Leite foi contemplada com um bolsa de estudos, nível doutoramento, pela Coordenação de Aperfeiçoamento de Pessoal de Nível Superior (CAPES; código financeiro 001).

7. Costa CS, Del-Ponte B, Assunção MCF, Santos IS. Consumption of ultra-processed foods and body fat during childhood and adolescence: a systematic review. Public Health Nutr 2018; 21:148-59.

8. Melo B, Rezende L, Machado P, Gouveia N, Levy R. Associations of ultra-processed food and drink products with asthma and wheezing among Brazilian adolescents. Pediatr Allergy Immunol 2018; 29:504-11.

9. Haddad MR, Sarti FM. Sociodemographic determinants of health behaviors among Brazilian adolescents: trends in physical activity and food consumption, 2009-2015. Appetite 2020; 144:104454

10. Ricardo CZ, Azeredo CM, Rezende LFM, Levy $\mathrm{RB}$. Co-occurrence and clustering of the four major non-communicable disease risk factors in Brazilian adolescents: analysis of a national school-based survey. PLoS One 2019; 14:e0219370.

11. Mozaffarian D, Afshin A, Benowitz NL, Bittner V, Daniels SR, Franch HA, et al. Population approaches to improve diet, physical activity, and smoking habits: a scientific statement from the American Heart Association. Circulation 2012; 126:1514-63. 
12. Micha R, Karageorgou D, Bakogianni I, Trichia E, Whitsel LP, Story M, et al. Effectiveness of school food environment policies on children's dietary behaviors: A systematic review and meta-analysis. PLoS One 2018; 13:e0194555.

13. Food and Agriculture Organization of the United Nations. School food and nutrition framework. Rome: Food and Agriculture Organization of the United Nations; 2019.

14. Noll PRES, Noll M, Abreu LC, Baracat EC, Silveira EA, Sorpreso ICE. Ultra-processed food consumption by Brazilian adolescents in cafeterias and school meals. Sci Rep 2019; 9:7162.

15. Azeredo CM, Rezende, LFM, Canella DS, Claro RM, Peres MFT, Luiz OC, et al. Food environments in schools and in the immediate vicinity are associated with unhealthy food consumption among Brazilian adolescents. Prev Med 2016; 88:73-9.

16. Godin KM, Chacón V, Barnoya J, Leatherdale ST. The school environment and sugar-sweetened beverage consumption among Guatemalan adolescents. Public Health Nutr.2017; 20:2980-7.

17. Rathi N, Riddell L, Worsley A. What influences urban Indian secondary school students' food consumption? - a qualitative study. Appetite 2016; 105:790-7.

18. Martinez-Ospina A, Sudfeld CR, González SA, Sarmiento OL. School food environment, food consumption, and indicators of adiposity among students 7-14 years in Bogotá, Colombia. J Sch Health 2019; 89:200-9.

19. Brasil. Lei no 11.947, de 16 de junho de 2009. Dispõe sobre o atendimento da alimentação escolar e do Programa Dinheiro Direto na Escola aos alunos da educação básica; altera as Leis no 10.880 , de 9 de junho de 2004, 11.273, de 6 de fevereiro de 2006, 11.507, de 20 de julho de 2007; revoga dispositivos da Medida Provisória no 2.178-36, de 24 de agosto de 2001, e a Lei no 8.913, de 12 de julho de 1994; e dá outras providências. Diário Oficial da União 2009; 17 jun.

20. Secretaria de Atenção Primária à Saúde, Ministério da Saúde. Sobre o Programa Saúde na Escola (PSE). https://aps.saude.gov.br/ape/pse (acessado em 31/Mai/2020).

21. Carmo AS, Assis MM, Cunha CF, Oliveira TRPR, Mendes LL, Carmo AS, et al. The food environment of Brazilian public and private schools. Cad Saúde Pública 2018; 34:e0014918.

22. Azeredo CM, Leite MA, Rauber F, Ricardo CZ, Levy RB. Are laws restricting soft drinks sales in Brazilian schools able to lower their availability? Rev Saúde Pública 2020; 54:42.

23. Instituto Nacional de Estudos e Pesquisas Educacionais Anísio Teixeira. Educação infantil. http://portal.inep.gov.br/educacao-infan til/ (acessado em 27/Dez/2019).

24. United Nations. Designing household survey samples: practical guidelines. New York: United Nations; 2005.
25. Peres MFT, Eisner M, Loch AP, Nascimento A, Papa CHG, Azeredo CM, et al. Violência, bullying e repercussões na saúde: resultados do Projeto São Paulo para o desenvolvimento social de crianças e adolescentes (SP-Proso). São Paulo: Departamento de Medicina Preventiva, Faculdade de Medicina, Universidade de São Paulo; 2018.

26. Instituto Brasileiro de Geografia e Estatística. Pesquisa Nacional de Saúde do Escolar - 2012. Rio de Janeiro: Instituto Brasileiro de Geografia e Estatística; 2013.

27. Instituto Brasileiro de Geografia e Estatística. Pesquisa Nacional de Saúde do Escolar - 2015. Rio de Janeiro: Instituto Brasileiro de Geografia e Estatística; 2016.

28. Tavares LF, Castro IRR, Levy RB, Cardoso LO, Passos MD, Brito FSB. Validade relativa de indicadores de práticas alimentares da Pesquisa Nacional de Saúde do Escolar entre adolescentes do Rio de Janeiro. Cad Saúde Pública 2014; 30:1029-41.

29. Levy RB, Castro IRR, Cardoso LO, Tavares LF, Sardinha LMV, Gomes FS, et al. Consumo e comportamento alimentar entre adolescentes brasileiros: Pesquisa Nacional de Saúde do Escolar (PeNSE), 2009. Ciênc Saúde Colet 2010; 15 Suppl 2:3085-97.

30. Diez-Roux AV. Multilevel analysis in public health research. Annu Rev Public Health 2000; 21:171-92.

31. Azeredo CM, Rezende LFM, Canella DS, Claro RM, Castro IRR, Carmo Luiz O, et al. Dietary intake of Brazilian adolescents. Public Health Nutr 2015; 18:1215-24.

32. Royston P, White IR. Multiple imputation by chained equations (MICE): implementation in stata. Journal of Statistical Software 2011; 45:1-20.

33. Godin KM, Chaurasia A, Hammond D, Leatherdale ST. Food purchasing behaviors and sugar-sweetened beverage consumption among Canadian secondary school students in the COMPASS study. J Nutr Educ Behav 2018; 50:803-12.

34. Clinton-McHarg T, Janssen L, Delaney $\mathrm{T}$, Reilly K, Regan T, Nathan N, et al. Availability of food and beverage items on school canteen menus and association with items purchased by children of primary-school age. Public Health Nutr 2018; 21:2907-14.

35. Calvert S, Dempsey RC, Povey R. Delivering in-school interventions to improve dietary behaviours amongst 11-to 16-year-olds: a systematic review. Obes Rev 2019; 20:543-53.

36. Boing AF, Subramanian SV, Boing AC. How important is school environment in explaining individual variance of health behaviors? Rev Saúde Pública 2019; 53:102. 
37. Coordenadoria de Ensino da Região Metropolitana da Grande São Paulo. Portaria Conjunta COGSP/CEI/DSE, de 23 de março de 2005. Normas para funcionamento de cantinas escolares. Diário Oficial do Estado de São Paulo 2005; 24 mar.

38. Secretaria Municipal de Educação. Portaria no 11 , de 15 de fevereiro de 2001. Dispõe sobre a proibição de comércio e venda de alimentos aos alunos da Rede Municipal de Ensino, e dá outras providências. Diário Oficial do Município de São Paulo 2001; 15 fev.
39. Coordenação Técnica de Alimentação e Nutrição, Coordenação Geral do Programa de Alimentação Escolar, Diretoria de Ações de Assistência Educacional, Fundo Nacional de Desenvolvimento da Educação. Ministério da Educação. Nota técnica no 02/2012. Regulamentação de cantinas escolares em escolas públicas do Brasil. Brasília: Ministério da Edução; 2012.

40. Observatório de Plano Nacional de Educação. Ensino Fundamental. https://www.observa toriodopne.org.br/home (acessado em 01/ Jun/2020). 


\section{Abstract}

Ultra-processed foods are markers of unhealthy eating patterns, and their consumption is high among adolescents. Characteristics of the school eating environment associate with student eating practices. This study aims to investigate the association between the presence of school canteens and the availability of ultra-processed foods in school canteens with the consumption of these foods, inside the school, among adolescents of the 9 th grade (14 years) of the public and private schools of the city of São Paulo, Brazil. We conducted a cross-sectional study with SP-Proso data, in a sample of 2,680 adolescents. We elaborated multilevel linear regression models to evaluate associations between the exposures: presence of school canteens and availability of ultra-processed foods with outcomes of consumption frequency of ultra-processed foods in the school. We found an association between the presence of school canteens and the higher frequency of consumption of sausages (0.46; 95\%CI: 0.24; 0.68), package snacks (0.50; 95\% CI: 0.19; 0.80), goodies (0.82; 95\% CI: $0.55 ; 1.09)$, and sugary drinks (0.34; 95\%CI: 0.06; 0.62 ), as well as a score of consumption frequency of ultra-processed foods (2.37; 95\%CI: 1.25; 3.48). The availability of package snacks, goodies, and sugary drinks in school canteens increased the frequency of consumption of these foods. We observed a dose-response effect between the diversity of ultra-processed foods in canteens and the frequency of consumption of these foods. A school food environment with greater availability of ultra-processed products is associated with their higher consumption in school, indicating the need to regulate the sale of food within these institutions.

School Feeding; Adolescent; Multilevel Analysis

\section{Resumen}

Los alimentos ultraprocesados son marcadores de patrones alimentarios no saludables y su consumo es elevado entre los adolescentes. Características del entorno alimentario escolar se asocian a prácticas alimentarias de estudiantes. El objetivo del estudio fue investigar la asociación entre la presencia de cantinas, y la disponibilidad de alimentos ultraprocesados en las mismas, con el consumo de estos alimentos dentro de la escuela, entre adolescentes del 9o año de la enseñanza fundamental de las redes pública y privada del Municipio de São Paulo, Brasil. Se realizó un estudio transversal con datos del SP-Proso, en una muestra de 2.680 adolescentes. Se realizaron modelos de regresión lineal multinivel para evaluar las asociaciones entre las exposiciones presencia de cantinas en las escuelas y disponibilidad de alimentos ultraprocesados con resultados de frecuencia de consumo de ultraprocesados en la escuela. La presencia de cantinas estuvo asociada a una mayor frecuencia de consumo de embutidos (0,46; IC95\%: 0,24; 0,68), aperitivos de bolsa (0,50; IC95\%: 0, 19; 0,80), golosinas (0,82; IC95\%: 0,55; 1,09) y bebidas azucaradas (0,34; IC95\%: 0,06; 0,62), así como un marcador de frecuencia de consumo de ultraprocesados (2,37; IC95\%: 1,25; 3,48). La disponibilidad de aperitivos de bolsa, golosinas y bebidas azucaradas en las cantinas aumentó la frecuencia de consumo de esos alimentos. Se observó un efecto dosis/ respuesta entre la diversidad de ultraprocesados en las cantinas y la frecuencia de consumo de estos alimentos. Un ambiente alimentario escolar con mayor disponibilidad de ultraprocesados está asociado a un mayor consumo de los mismos en la escuela, lo que apunta a la necesidad de regular el comercio de alimentos dentro de estas instituciones.

Alimentación Escolar; Adolescente; Análisis Multinivel
Recebido em 10/Jun/2020

Versão final representada em 18/Ago/2020

Aprovado em 04/Set/2020 